\title{
Biomechanical Properties of a New Anatomical Locking Metal Block Plate for Opening Wedge High Tibial Osteotomy: Uniplane Osteotomy
}

\author{
Seung-Beom Han, $\mathrm{MD}^{1}$, Ji-Hoon Bae, $\mathrm{MD}^{2}$, Sung-Jae Lee, $\mathrm{MD}^{3}$, Tae-Gon Jung, $\mathrm{MD}^{3}$, Kang-Hee Kim, $\mathrm{MD}^{3}$, \\ Jae Ho Kwon, $\mathrm{MD}^{4}$, and Kyung Wook Nha, $\mathrm{MD}^{4}$ \\ ${ }^{1}$ Department of Orthopaedic Surgery, Korea University Anam Hospital, Seoul; ${ }^{2}$ Department of Orthopaedic Surgery, Korea University Ansan Hospital, Ansan; \\ ${ }^{3}$ Department of Biomedical Engineering, Inje University, Gimhae; ${ }^{4}$ Department of Orthopaedic Surgery, Inje University Ilsan Paik Hospital, Ilsan, Korea
}

\begin{abstract}
Purpose: The purpose of this study was to evaluate the biomechanical properties of a new anatomical locking metal block plate by comparing the initial biomechanical stability of three different fixation constructs for open wedge high tibial osteotomy (HTO).

Materials and Methods: Sawbones composite tibiae were used to make a 10-mm opening osteotomy model with uniplane technique. The osteotomy was secured with three different types of plates: Group I, new osteotomy plate without a metal block (n=5); Group II, new osteotomy plate with a 10mm metal block $(n=5)$; and Group III, two short metal block plates $(n=5)$. Single load to failure test and staircase load-controlled cyclical failure test were performed. In the single load to failure test, the yield load, maximum failure load, and the displacement of the osteotomy gap were measured. In the staircase cyclical load to failure test, the total number of cycles to failure was recorded. Failure modes were observed during both single and cyclic load tests.

Results: Group II showed the highest yield and ultimate loads ( $1829 \pm 319$ N, 3493 \pm 1250 N) compared to Group I (1512 \pm 157 N, $2422 \pm 769$ N) and Group III $(1369 \pm 378 \mathrm{~N}, 2157 \pm 210 \mathrm{~N}, \mathrm{p}<0.05)$. The displacement of the opening gap in Group II $(0.34 \pm 0.35 \mathrm{~mm})$ was significantly lesser than the other groups $(\mathrm{p}<0.05)$. In the staircase cyclical load to failure test, the total number of cycles to failure was 12,860 at $950 \mathrm{~N}$ in Group III, 20,280 at 1,140 $\mathrm{N}$ in Group I, and 42,816 at 1,330 N in Group II ( $\mathrm{p}<0.05)$. All the specimens showed complete fracture of the intact lateral sawbones area and slight displacement of the distal fragment of the specimens in the single load to test. None of the specimens showed deformed or broken screws and plates during the single load to test. During the fatigue test with staircase cyclic loading, no fracture of the lateral sawbones area was observed.

Conclusions: This study demonstrated that the new anatomical locking metal block plate could provide sufficient primary stability for open wedge HTO. The addition of a metal block to this new plate can increase the stability of the osteotomy compared to the one without a metal block.
\end{abstract}

Keywords: Knee, High tibial osteotomy, Anatomical locking metal block plate, Stability

\section{Introduction}

High tibial osteotomy (HTO) is a surgical treatment option for

Received January 17, 2014; Revised (1st) April 7, 2014;

(2nd) June 19, 2014; Accepted June 20, 2014

Correspondence to: Kyung Wook Nha, MD

Department of Orthopaedic Surgery, Inje University Ilsan Paik Hospital,

170 Juhwa-ro, Ilsanseo-gu, Goyang 411-706, Korea

Tel: +82-31-910-7301, Fax: +82-31-910-7967

E-mail:kwnhamj@hotmail.com

This is an Open Access article distributed under the terms of the Creative Commons Attribution Non-Commercial License (http://creativecommons.org/licenses/by-nc/3.0/) which permits unrestricted non-commercial use, distribution, and reproduction in any medium, provided the original work is properly cited. symptomatic medial compartment osteoarthritis of the knee with varus deformity ${ }^{1)}$. Currently, medial open wedge osteotomy is popular due to its several advantages over lateral closing wedge osteotomy, such as easy approach, low incidence of peroneal nerve injury, and preservation of the proximal bone. The initial fixation strength of fixatives for open wedge osteotomy and maintenance of its stability until complete healing of the opening gap are very important for not only successful clinical results but also the healing of osteotomy. Many fixatives with a combination of short or long, locked or unlocked, and with or without a metal block (rectangular or tapered) have been introduced for medial open wedge HTO. Of those, the TomoFix plate (TomoFix Osteotomy System; DePuy Synthesis, West Chester, PA, USA) has been widely used due to its superior biomechanical properties compared to other 
fixatives ${ }^{2-4}$. Several authors have recently reported satisfactory clinical results of HTO using the TomoFix plate ${ }^{5,6}$.

Although the TomoFix plate provides biomechanically stable fixation, it is too large or too long for Asian patients, which results in local irritation and no purchase of D-hole. Especially, local irritation has been a major complaint in many patients after HTO using the plate ${ }^{7-9)}$. Short spacer plates such as the Tibial Opening Wedge Osteotomy Plate, known as the Puddu plate, (Arthrex Inc., Naples, FL, USA), and the Aescula plate (Medyssey, Uijeongbu, Korea) have low incidence of local irritation, but several authors have reported on the high risk of plate-related complications ${ }^{10-13)}$. To overcome these limitations, we developed a low profile, anatomical locking metal block plate for medial open wedge HTO. The advantages of both long locking plates and short metal block plates are incorporated into the new plate.

The purposes of this study was to evaluate the biomechanical properties of the new plate based on the comparison of three different fixation constructs in terms of the initial mechanical stability in a medial open wedge high tibial uniplane osteotomy model. Our hypothesis was that the new anatomical locking metal block plate with a metal block would be superior to other fixation constructs regarding both initial fixation strength and resistance to displacement of the opening gap.

\section{Materials and Methods}

\section{Plate Design}

The new anatomical locking metal block plate (OhtoFix;
Ohtomedical Co. Ltd., Goyang, Korea) is specifically designed for medial open wedge high tibial uniplane osteotomy (Fig. 1). This plate is anatomically pre-contoured to fit the anteromedial proximal tibia. The upper end of the plate has a 5-degree posterior slope for better placement onto the proximal tibia. The plate has six holes for fixed angle locking screws, three in the upper end and the other three in the shaft. In the upper part of the plate shaft corresponding to the osteotomy level, there is a sliding hole for a metal block. The shape of the sliding hole is oblong to adjust to the location of the metal block depending on the osteotomy site. The metal block is wedge-shaped. The anterior height of the metal block is two thirds of the posterior height to create a wedge-shape opening gap for preventing any change in the posterior slope of the proximal tibia. The metal block is available in different sizes corresponding to the amount of opening osteotomy $(8,10,12,14$ and $16 \mathrm{~mm})$. The plate is made of commercially available titanium-alloy.

\section{Medial Open Wedge Osteotomy Model Preparation}

Large composite left tibiae with 10 degree varus of the proximal tibia ( $n=15$, 4th generation; Pacific Research Laboratories, Vashon Island, WA, USA) were used as specimens. The composite tibia has been known to have the structural properties of a healthy adult tibia. All osteotomies were performed in the same manner by a single experienced surgeon. Under fluoroscopic imaging, a guide pin was drilled across the proximal end of the tibia in a medial to lateral direction. The guide pin was positioned at the level of the superior aspect of the tibial tubercle (approximately

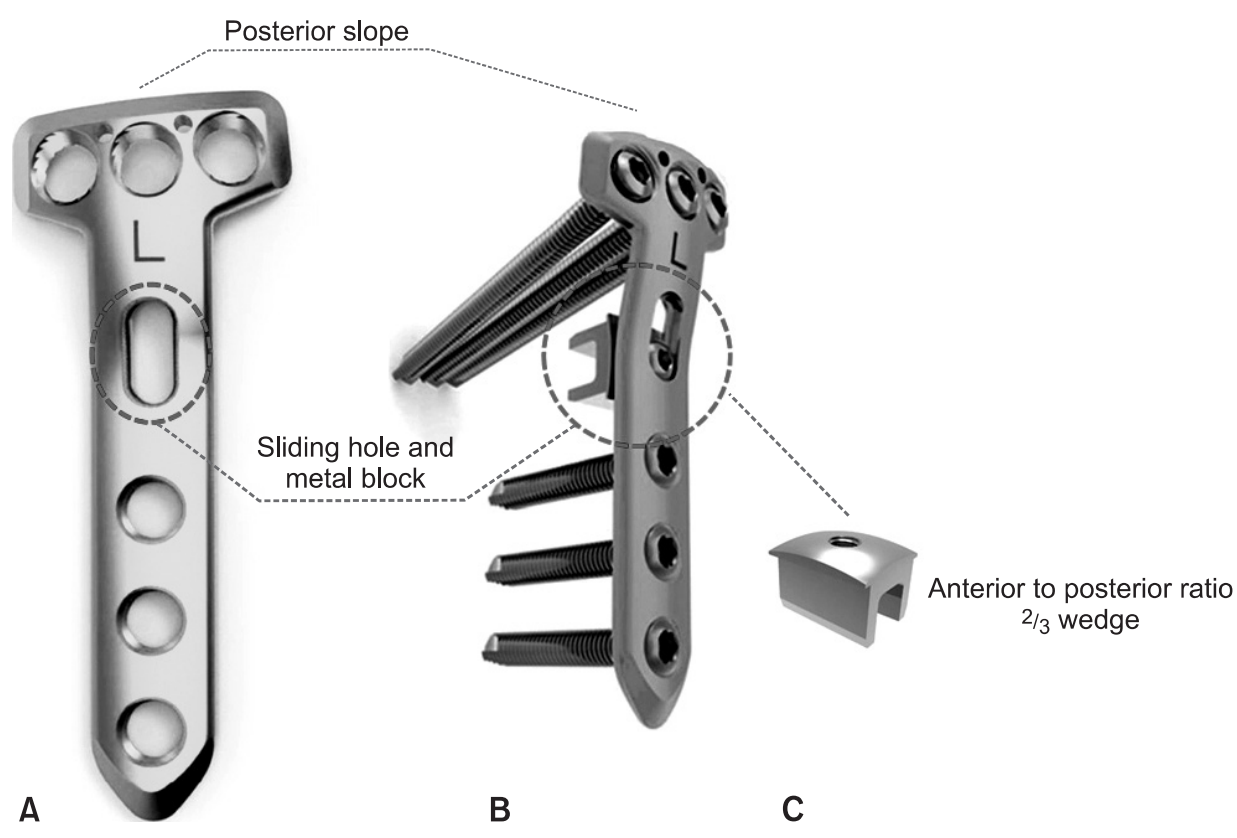

Fig. 1. The new anatomical locking metal block plate. (A) The upper end of the plate has a 5-degree posterior slope for better placement onto the proximal tibia. (B) The shape of the sliding hole is oblong to adjust to the location of a metal block depending on the osteotomy site. (C) The anterior height of the metal block is two thirds of the posterior height to create a wedge-shaped opening gap. L: plate for the left knee. 
30-mm distal to the medial tibial plateau) and oriented obliquely to the end, approximately $1 \mathrm{~cm}$ below the joint line at the posterolateral tibial cortex ${ }^{14)}$. A cortical cut was then made using an oscillating saw for the anterolateral, medial, and posterior cortices, and an osteotome chisel was advanced to within $1 \mathrm{~cm}$ of the posterolateral edge of the tibia, leaving the lateral cortex intact. Once the osteotomy had been completed, the osteotomy surface was opened with a gap spreader to obtain a 10-mm opening gap. A 10-mm wedge shaped metal block was inserted to maintain the opening gap until completing plate fixation. After the metal block insertion, the osteotomy gap was gently compressed to be wedgeshaped according to the shape of the metal block to prevent any change in the posterior slope of the proximal tibia.

\section{Study Groups}

After the 10-mm opening gap osteotomy was completed, the osteotomy was secured with three different types of plates according to study group (Fig. 2): Group I, the new osteotomy plate without a metal block ( $\mathrm{n}=5)$; Group II, the new osteotomy plate with a 10-mm metal block ( $\mathrm{n}=5$ ); and Group III, two short spacer plates ( $n=5$, Aescula plate). In Group I, the plate was placed in the same location as in Group II. The hole for the metal block was centered over the osteotomy gap with three horizontal screws placed proximally and three screws distally. When all six screws were inserted, the metal block was removed, and then all six screws were locked using a torque-limiting driver. In Group II, all the procedures for securing the plate were the same as in
Group I, except for leaving the metal block attached to the plate. After locking the screws, the metal block in the opening gap was attached to the plate with a 3.5-mm screw. The length of locking screws in each corresponding hole was the same as in Group I and Group II. All the screws used in Groups I and II were bicortical. In Group III, two short metal block plates (Aescula plate) were secured: one with a 6-mm metal block was placed at the anterior one third of the osteotomy gap and the other with a 10mm metal block, at the posterior one third of the osteotomy gap. All the screws were unicortical in Group III.

\section{Biomechanical Testing \\ 1) Set-up for compression loading}

The compression loading axis was located to be identical with the center of the anteroposterior axis and $65 \%$ of the tibial plateau (medial end of the tibial plateau, $0 \%$ and lateral end of the tibial plateau, $100 \%$ in three specimens in each group by using the vernier calipers. Next, the proximal and distal ends of the specimens were embedded in polyurethane casting resin and mounted in a specially designed fixture that was connected to an MTS machine (MTS 858 Bionix; MTS System Corp., Eden Prairie, MN, USA) after contact with the load axis and the actuator (Fig. 3). The orientation of the compression loading axis was vertical to the ground.

2) Set-up for displacement measurements

Two markers ( $\phi=0.5 \mathrm{~mm}$; stainless steel, pin type) were attached
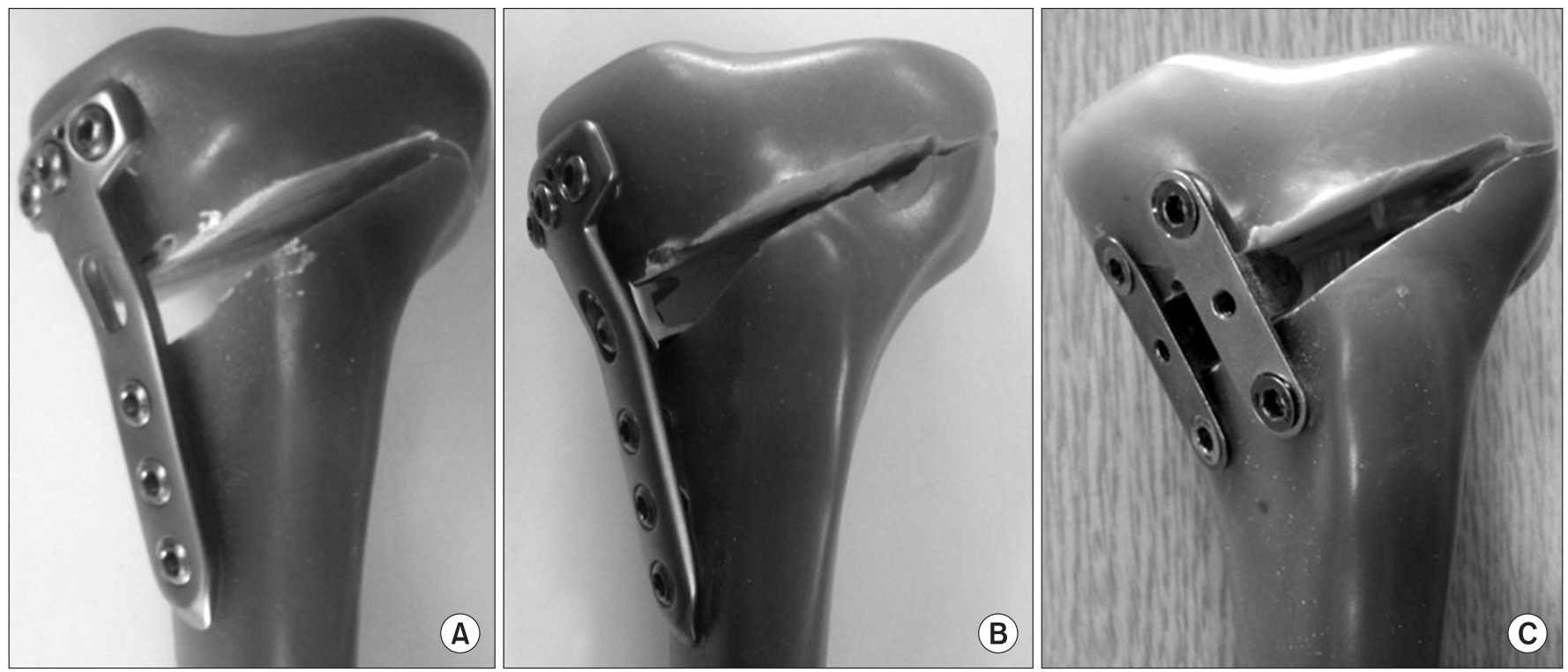

Fig. 2. Three different types of plates are used in sawbones tibia models: (A) Group I, new osteotomy plate without a metal block; (B) Group II, new osteotomy plate with a 10-mm metal block; and (C) Group III, two short spacer plates. 
A

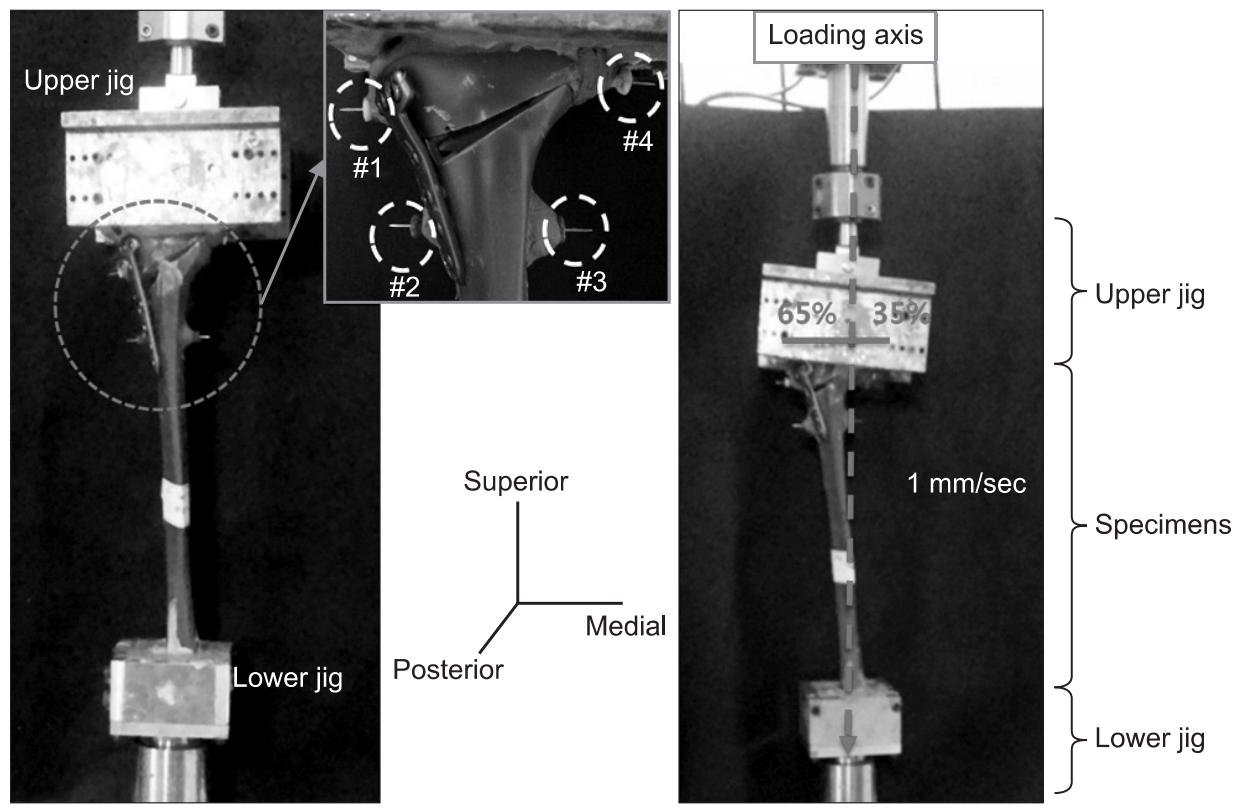

B

Fig. 3. (A) In the biomechanical testing setup, the sawbones composite tibia is mounted in a specially designed fixture connected to an MTS machine. (B) Compression loading axis test set-up is created. to the medial cortex near the superior and inferior tips of the wedge gap opening. Three-dimensional (3D) movements of the gap before and after loading sequences were assessed in a stereophotogrammetry setup with two digital cameras (IXUS 200IS; Cannon, Tokyo, Japan). This is a method capable of determining $3 \mathrm{D}$ coordinates of the markers ( $\phi=0.5 \mathrm{~mm}$; stainless steel, pin type) in space. It uses the method of direct linear transformation and stereophotogrammetry based on two images of the markers that were taken simultaneously by two cameras. Here, the two digital cameras were oriented at 60 degrees to the object (i.e., markers) to form an isosceles triangle. The laboratory coordinate system was established through a calibration procedure with a specially-constructed calibration frame. The details of the calibration procedure can be found in the literature ${ }^{15)}$. The $3 \mathrm{D}$ movement of the markers, or the gap between the markers, was assessed based on changes in the 3D coordinates of the markers before and after loading sequences (resolution, $0.01 \mathrm{~mm}$ ).

\section{Mechanical Testing}

To precondition the specimens, pre-cyclic loads (range, 50 to $800 \mathrm{~N} ; 60$ cycles, $0.25 \mathrm{~Hz}$ ) were applied before all experiments ${ }^{3)}$. Then, compression loading to failure $(1 \mathrm{~mm} / \mathrm{sec})$ was applied at the location of $65 \%$ of the tibial plateau (medial end of the tibial plateau, $0 \%$ and lateral end of the tibial plateau, 100\%) in three specimens in each group. The yield load was defined as the point at which the first reduction in loading occurs in the loaddisplacement curve and the ultimate load as the maximum loading. In the same fashion, dynamic fatigue load (20,000 cycles, 2
$\mathrm{Hz}$ ) was applied to simulate 12 weeks postoperative duration in a staircase loading sequence until failure, initially from $50 \%$ of the maximum failure load in an increment of $10 \%$ in two specimens in each group ${ }^{3,16)}$. Loading was terminated when actuator displacement of more than $2 \mathrm{~mm}$ or a breakage of fixatives were observed during one loading cycle, which was defined as the failure of the construct. The total number of cycles and loading steps were recorded. Displacement across the opening gap until failure was obtained at an interval of $20 \mathrm{~Hz}$ in all experiments. The mechanism of failure mode for each test was also recorded. The yield load, ultimate load, number of cycles to failure under cyclic loading, and displacement of medial opening gap of three different fixation types of plates were compared. Statistical analysis was performed using an analysis of variance test (SPSS ver. 12.0; SPSS Inc., Chicago, IL, USA). Statistical significance was set at $\mathrm{p}<0.05$.

\section{Results}

\section{Single Load to Failure Test}

All specimens in each group tolerated axial cyclic loading of $150 \mathrm{~N}$ and $800 \mathrm{~N}$ without failure. Group II (OhtoFix with a 10$\mathrm{mm}$ metal block) showed significantly high yield and ultimate load $(1829 \pm 319 \mathrm{~N}$ and $3493 \pm 1250 \mathrm{~N}$, respectively; $\mathrm{p}=0.031$ and $\mathrm{p}=0.029$, respectively) compared to Group I $(1512 \pm 157 \mathrm{~N}$ and $2422 \pm 769 \mathrm{~N}$, respectively) and Group III $(1369 \pm 378 \mathrm{~N}$ and $2157 \pm 210 \mathrm{~N}$, respectively) (Fig. 4). There were no significant differences between Group I and Group III. The use of a metal block in Group II resulted in consistently higher fixation strength and 


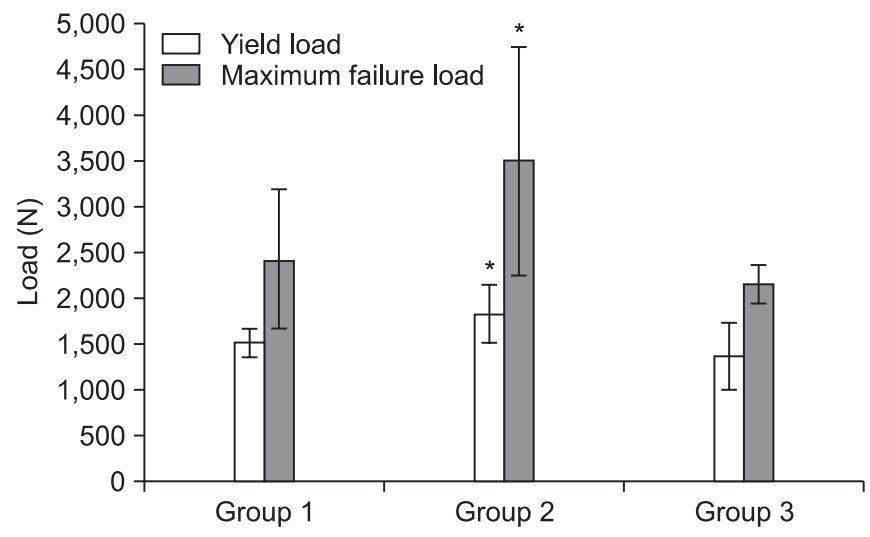

Fig. 4. The bar graph shows the averages of the yield load and maximum failure load. Group 1: new osteotomy plate without a metal block, Group 2: new osteotomy plate with a 10-mm metal block, Group 3: two short spacer plates. ${ }^{*} \mathrm{p}<0.05$, compared to either group 1 or group 3.

improved load bearing capabilities compared to the one without a metal block in Group I. Similarly, the least medial opening gap displacement was observed in Group II $(0.34 \pm 0.35 \mathrm{~mm})$ followed by Group I $(0.65 \pm 0.07 \mathrm{~mm})$ and Group III $(1.93 \pm 1.61 \mathrm{~mm})$ $(\mathrm{p}=0.042)$ (Fig. 5).

\section{Fatigue Test with Staircase Cyclic Loading}

In the staircase long-term cyclic loading test, the two short metal block plate group (Group III) failed first at $950 \mathrm{~N}$ (50\% of the average maximum failure load). The new anatomical locking metal block plate without a metal block group (Group I) failed at $1,140 \mathrm{~N}$ ( $60 \%$ of the average maximum failure load) and the new anatomical locking metal block plate with a $10-\mathrm{mm}$ metal block group (Group II) failed at $1,330 \mathrm{~N}$ ( $70 \%$ of the average maximum failure load) (Table 1).

\section{Failure Modes}

In the single load to failure test, all the specimens showed the same failure mode. A crack first developed in the intact lateral sawbones area (lateral cortex of the proximal tibia) and it extended to the end of the lateral side, eventually resulting in complete fracture with slight displacement of the distal fragment of the specimens. None of the specimens showed deformed or broken screws and plates in the single load to failure test. In the fatigue test with staircase cyclic loading, no fracture of the lateral sawbones area was observed.

\section{Discussion}

The present biomechanical study confirmed our hypothesis that initial fixation strength of the new anatomical locking plate with

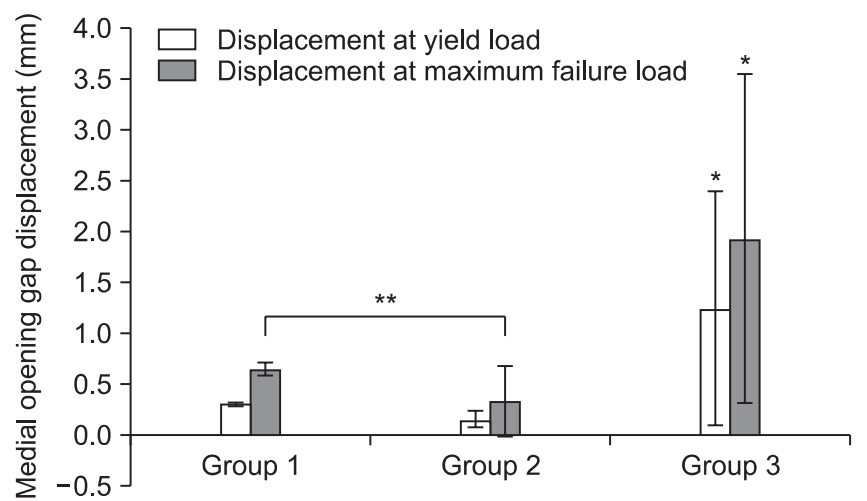

Fig. 5. The graph shows the average displacement of the medial opening gap in the single-load test. Group 1: new osteotomy plate without a metal block, Group 2: new osteotomy plate with a 10-mm metal block, Group 3: two short spacer plates. ${ }^{*} \mathrm{p}<0.05$, compared to either group 1 or group $2,{ }^{* *} \mathrm{p}<0.05$, comparison between group 1 and group 2 .

Table 1. Cyclic Loading to Failure

\begin{tabular}{llll}
\hline Variable & \multicolumn{1}{c}{$950 \mathrm{~N}^{\mathrm{a})}$} & $1,140 \mathrm{~N}^{\mathrm{b})}$ & $1,330 \mathrm{~N}^{\mathrm{c})}$ \\
\hline Group 1 & No failure & 20,280 cycles & \\
Group 2 & No failure & No failure & 42,816 cycles \\
Group 3 & 12,860 cycles & & \\
\hline${ }^{\text {a) } \text { b) }^{\text {b) }} \text {, and }}{ }^{\text {c) }}$ are $50 \%, 60 \%$, and $70 \%$ of the average maximum failure load \\
of all specimens, respectively. &
\end{tabular}

a metal block would be superior to that of other fixation constructs. In addition, we found that initial fixation strength of the new anatomical locking plate with a metal block was comparable to the previously reported strength of the TomoFix plate ${ }^{3)}$. The addition of a metal block provided increased primary mechanical stability compared to that with the new anatomical locking plate alone, which supports the clinical use of a metal block for the augmentation of mechanical strength in cases of large amount of correction.

The average ultimate failure load of the new anatomical locking metal block plate alone in the single load to failure test was $2422 \pm 769 \mathrm{~N}$, which is close to the axial compressive force to an adult tibiofemoral joint during level walking ${ }^{17}$. This data supports that this new anatomical locking metal block plate has sufficient strength to bear load during daily walking and would be able to maintain correction until complete healing of the osteotomy site. Moreover, the addition of a 10-mm metal block to the new anatomical locking metal block plate provided a higher ultimate failure load $(3493 \pm 1250 \mathrm{~N})$. Our data are comparable indirectly to the previous biomechanical studies. Agneskirchner et al. ${ }^{3)}$ compared the biomechanical properties of four different fixation systems in a 10-mm opening HTO model using third generation 
synthetic composite tibiae. They reported that the ultimate failure load of the TomoFix plate in a single load to failure test was an average of $2881 \mathrm{~N}$. Stoffel et al. ${ }^{4)}$ reported the ultimate failure load of the Puddu plate and TomoFix was an average of $2537 \mathrm{~N}$ and $2904 \mathrm{~N}$, respectively, in a 15-mm opening HTO model using synthetic composite tibiae. We found that the new anatomical locking metal block plate alone has a slightly lower ultimate failure load than that of the TomoFix plate; however, the addition of a metal block to the new plate exceeded the ultimate failure load of the TomoFix plate. Therefore, the use of a metal block can be taken into consideration to augment stability of the osteotomy gap depending on the patient's body weight and amount of correction.

Progressive loss of correction is one of the serious complications after medial open wedge HTO. Appropriate surgical techniques with sufficient strength of fixatives are necessary. The fatigue strength of a fixative is important to prevent progressive varus collapse of osteotomy in early postoperative periods. Axial load-controlled cyclical staircase loading tests have been often used to test the fatigue strength of fixatives ${ }^{3,18,19)}$. These loading tests are known to simulate in vivo physiologic conditions. Based on the definition of failure of constructs (more than $2 \mathrm{~mm}$ gap displacement or a breakage of fixatives), we observed that all the constructs with fixatives tolerated well under the short-term cyclic loads but they had different behavior under the long-term cyclic loads. The construct with new anatomical locking metal block plates with a 10-mm metal block resisted more than three times and twice the amount of loading cycles when compared to the short metal block plates group and the new plate without a metal block group, respectively. This finding supports the results of a previous study ${ }^{3}$, which showed that the construct with a long rigid locking plate resisted more loading cycles than the short metal block plates. In addition, the construct with the new anatomical locking metal block plate and a $10-\mathrm{mm}$ metal block has a fatigue strength comparable to that of the TomoFix ${ }^{3}$. This suggests that the new anatomical locking metal block plate provides stable fixation for early weight-bearing. However, weight-bearing should be delayed if short metal block plates are used.

Insufficient strength of fixatives can result in collapse of an opening gap in the early postoperative periods. Significant amount of collapse of an opening gap requires re-varization of the lower leg. During both the single load to failure test and fatigue test, the lowest displacement of the opening gap was observed in the group of a new plate with a metal block and the highest displacement, in the group of two short metal block plates. This suggests that the length of a plate, number of screws, and the use of unlocked or locked screws can affect the fixative stability. However, Agneskirchner et al. ${ }^{3)}$ reported that short spacer plates provided better stability than the prototype long spacer plate, suggesting the length of the implant does not solely determine the implant stability regarding displacement. We are not able to compare the results of gap displacement with previous studies due to the different designs of the metal block plates and, therefore, we cannot make conclusions on the effect of the length of the metal block plate on gap displacement.

There are several limitations of this study. First, the results of our study can be applied only to an osteotomy with a $10-\mathrm{mm}$ opening gap and the time zero point of initial fixation. Therefore, our results do not reflect the osteotomy with different sizes of opening gaps and the biomechanical properties at multiple time points. Second, the screw fixation method used in Groups I and II may differ from in vivo surgical technique. We used bicortical screws both in Groups I and II to ensure equal experimental conditions between the groups. However, in most clinical cases, proximal horizontal locking screws are unicortical. This may result in different biomechanical properties. In addition, it might not have been appropriate to compare the biomechanical strength of nonlocking screws with unicortical fixation in Group III with that of locking screws with bicortical fixation in Groups I and II. Third, we used fourth generation composite synthetic sawbones tibiae for the biomechanical test to avoid inter-specimens variability. Therefore, the condition of soft tissues was not considered, which may affect the biomechanical stability of the osteotomy. Fourth, the failure mode during the single load to failure test was the fracture of the lateral part of the sawbones with slight displacement of the distal fragment. No broken or grossly deformed fixatives were observed during the test. However, failure modes may depend on different clinical situations. Fifth, it remains unclear whether this new plate can reduce soft tissue irritation; thus, further clinical studies are warranted. Sixth, we did not test or compare with many other fixation constructs. Finally, the number of specimens in each group may not be enough to achieve sufficient statistical power. In spite of these limitations of the study, we believe that this new anatomical locking metal block plate would be a reliable fixative, especially in Korean (Asian) patients.

\section{Conclusions}

The present biomechanical study demonstrated that the new anatomical locking metal block plate provides sufficient primary stability for medial open wedge HTO. The addition of a metal block to this new plate can increase the stability of the osteotomy 
compared to the one without a metal block.

\section{Conflict of Interest}

No potential conflict of interest relevant to this article was reported.

\section{Acknowledgements}

The authors would like to express special thanks to Dr. Guoan Li (Biomechanical laboratory of Harvard Medical School, Boston, MA, USA).

\section{References}

1. Lee DC, Byun SJ. High tibial osteotomy. Knee Surg Relat Res. 2012;24:61-9.

2. Raja Izaham RM, Abdul Kadir MR, Abdul Rashid AH, Hossain MG, Kamarul T. Finite element analysis of Puddu and Tomofix plate fixation for open wedge high tibial osteotomy. Injury. 2012;43:898-902.

3. Agneskirchner JD, Freiling D, Hurschler C, Lobenhoffer P. Primary stability of four different implants for opening wedge high tibial osteotomy. Knee Surg Sports Traumatol Arthrosc. 2006;14:291-300.

4. Stoffel K, Stachowiak G, Kuster M. Open wedge high tibial osteotomy: biomechanical investigation of the modified Arthrex Osteotomy Plate (Puddu Plate) and the TomoFix Plate. Clin Biomech (Bristol, Avon). 2004;19:944-50.

5. Floerkemeier S, Staubli AE, Schroeter S, Goldhahn S, Lobenhoffer P. Outcome after high tibial open-wedge osteotomy: a retrospective evaluation of 533 patients. Knee Surg Sports Traumatol Arthrosc. 2013;21:170-80.

6. Bode G, von Heyden J, Pestka J, Schmal H, Salzmann G, Sudkamp N, Niemeyer P. Prospective 5-year survival rate data following open-wedge valgus high tibial osteotomy. Knee Surg Sports Traumatol Arthrosc. 2013 Nov 19 [Epub]. http://dx.doi.org/10.1007/s00167-013-2762-y.

7. Niemeyer P, Schmal H, Hauschild O, von Heyden J, Sudkamp NP, Kostler W. Open-wedge osteotomy using an internal plate fixator in patients with medial-compartment gonarthritis and varus malalignment: 3-year results with regard to preoperative arthroscopic and radiographic findings. Arthroscopy. 2010;26:1607-16.
8. Valkering KP, van den Bekerom MP, Kappelhoff FM, Albers GH. Complications after tomofix medial opening wedge high tibial osteotomy. J Knee Surg. 2009;22:218-25.

9. Miller BS, Downie B, McDonough EB, Wojtys EM. Complications after medial opening wedge high tibial osteotomy. Arthroscopy. 2009;25:639-46.

10. Jung WH, Chun CW, Lee JH, Ha JH, Kim JH, Jeong JH. Comparative study of medial opening-wedge high tibial osteotomy using 2 different implants. Arthroscopy. 2013;29: 1063-71.

11. Schroter S, Gonser CE, Konstantinidis L, Helwig P, Albrecht D. High complication rate after biplanar open wedge high tibial osteotomy stabilized with a new spacer plate (Position HTO plate) without bone substitute. Arthroscopy. 2011;27: 644-52.

12. Nelissen EM, van Langelaan EJ, Nelissen RG. Stability of medial opening wedge high tibial osteotomy: a failure analysis. Int Orthop. 2010;34:217-23.

13. Spahn G. Complications in high tibial (medial opening wedge) osteotomy. Arch Orthop Trauma Surg. 2004;124:64953.

14. Hernigou P, Medevielle D, Debeyre J, Goutallier D. Proximal tibial osteotomy for osteoarthritis with varus deformity: a ten to thirteen-year follow-up study. J Bone Joint Surg Am. 1987;69:332-54.

15. Pourcelot P, Audigie F, Degueurce C, Geiger D, Denoix JM. A method to synchronise cameras using the direct linear transformation technique. J Biomech. 2000;33:1751-4.

16. Hoenig M, Gao F, Kinder J, Zhang LQ, Collinge C, Merk BR. Extra-articular distal tibia fractures: a mechanical evaluation of 4 different treatment methods. J Orthop Trauma. 2010;24: 30-5.

17. Kuster MS, Wood GA, Stachowiak GW, Gachter A. Joint load considerations in total knee replacement. J Bone Joint Surg Br. 1997;79:109-13.

18. Pape D, Lorbach O, Schmitz C, Busch LC, Van Giffen N, Seil R, Kohn DM. Effect of a biplanar osteotomy on primary stability following high tibial osteotomy: a biomechanical cadaver study. Knee Surg Sports Traumatol Arthrosc. 2010;18: 204-11.

19. Taylor WR, Heller MO, Bergmann G, Duda GN. Tibio-femoral loading during human gait and stair climbing. J Orthop Res. 2004;22:625-32. 